\title{
Holographic charm and bottom pentaquarks. III. Excitations through photoproduction of heavy mesons
}

\author{
Yizhuang Liu $\odot^{*}$ \\ Institute of Theoretical Physics, Jagiellonian University, 30-348 Krakw, Poland \\ Kiminad A. Mamo ${ }^{\dagger}$ \\ Physics Division, Argonne National Laboratory, Argonne, Illinois 60439, USA \\ Maciej A. Nowak \\ Institute of Theoretical Physics and Mark Kac Center for Complex Systems Research, Jagiellonian University, \\ 30-348 Krakw, Poland \\ Ismail Zahed ${ }^{\S}$ \\ Center for Nuclear Theory, Department of Physics and Astronomy, Stony Brook University, \\ Stony Brook, New York 11794-3800, USA
}

(Received 8 September 2021; accepted 6 November 2021; published 17 December 2021)

\begin{abstract}
We consider the photoexcitation of charm and bottom pentaquarks with the holographic assignments $\left[\frac{1}{2} \frac{1}{2}^{-}\right]_{S=0,1}$ and $\left[\frac{1}{2}^{-}\right)_{S=1}$, in the photoproduction of heavy vector mesons such as charmonia and bottomonia near threshold. We use a Witten diagram to combine the s-channel photoexcitation of holographic pentaquarks with a massive t-channel graviton or tensor glueball exchange, to extract the scattering amplitude for heavy meson photoproduction in the threshold region. The pentaquark signal is too weak to be detected at current electron facilities.
\end{abstract}

DOI: 10.1103/PhysRevD.104.114023

\section{INTRODUCTION}

The recently released data from the LHCb collaboration show the existence of three narrow pentaquark states $P_{c}(4312,4440,4457)$ [1]. This new high statistics data supersedes the previously reported $P_{c}(4450)$, and weakens the status of the previously reported $P_{c}(4380)$ [2]. This supports the holographic pentaquark construction by two of us where three degenerate pentaquarks $\left[\frac{1}{2} \frac{1}{2}^{-}\right]_{S=0,1}$ and $\left[\frac{1}{2} \frac{3}{2}^{-}\right]_{S=1}$ were predicted, in the triple limit of a large number of colors, a large 't Hooft coupling $\lambda$, and a heavy quark mass $[3,4]$. Similar assignments were subsequently made using the molecular construction [5-8].

The three holographic pentaquark states split when spinorbit interactions are considered $[9,10]$. The split states are not only close to the masses reported by LHCb, but their

\footnotetext{
yizhuang.liu@uj.edu.pl

kmamo@anl.gov

maciej.a.nowak@uj.edu.pl

§ismail.zahed@stonybrook.edu
}

Published by the American Physical Society under the terms of the Creative Commons Attribution 4.0 International license. Further distribution of this work must maintain attribution to the author(s) and the published article's title, journal citation, and DOI. Funded by SCOAP ${ }^{3}$. partial decay widths to a variety of open and hidden channels with heavy charmed mesons are also close to some of the newly reported widths by $\mathrm{LHCb}$ [1]. The holographic results extend to three new bottom pentaquark states. The newly reported and narrow $P_{c}(4337)$ at 3-sigma significance [11], is not supported by our holographic analysis of the low-lying pentaquark states. The even and odd parity excited holographic pentaquark states $P_{c}^{*}$ are heavier.

For completeness, we note that pentaquark states with hidden charm were suggested in [12,13] and analyzed by many [14-18] (and references therein). Given the closeness of the reported charmed pentaquarks to hadronic thresholds, most analyses suggest a molecular form [5,19-25]. Alternative constructions suggest solitonic molecules [26], string based molecules [20,27], and light cone based quarkonia [28].

The holographic charm (bottom) pentaquark states are bound topological molecules with hidden charm (bottom), essentially $\left[D, D^{*}\right]\left(\left[B, B^{*}\right]\right)$ mesons bound to a topological baryon at the boundary. They are free of some of the ambiguities related to the choice of meson exchanges and form factors $[12,21,29]$. The dual of the form factors is the instanton core which is fixed by gauge-gravity (mesonglueball) interactions in bulk. The dual of the meson exchanges are bulk light-light and heavy-light exchanges 
warped by the D-brane geometry, and the dual of the core gluon exchanges are bulk gravity and gravitons, in conformity with confinement, chiral symmetry, heavy quark symmetry, and vector dominance. The relativistic amplitudes Reggeize with proper crossing symmetries, as expected from a string based formulation. The construction involves very few parameters (the flavor brane tension, the Kaluza-Klein (KK)-scale and the mean heavy-light meson mass).

A more detailed way to understand the structure of the holographic pentaquarks, is through their strong two-body decay channels [10], or even better by directly producing them using photoproduction of charmonium (bottomonium) on the proton in the threshold region [30-32] as currently pursued at JLab [33]. Precision data are required to disentangle the s-channel production from the dominant t-channel contribution, in the nearly diffractive regime. The purpose of this paper is to address this issue, solely in the holographic construction whereby three degenerate pentaquark states emerge with specific spin-isospin-parity assignments and interactions.

The organization of this paper is as follows: in Sec. II we characterize the holographic transition form factor for the photoprocess $\gamma+p \rightarrow X$ for the three holographic pentaquarks with $X=P_{c, b}$. In the heavy quark limit, it is Pauli-like with the transition magnetic moment $\mu_{X} / \mu_{N} \sim$ $\left(m_{N} / m_{X}\right)^{\frac{3}{2}}$. The form factor for the decay process $X \rightarrow$ $V+p$ with $V=J / \Psi, \Upsilon$ is also discussed. In Sec. III we construct the s-channel photoproduction of charmonium (bottomium) by photoexcitation of a pentaquark state using a Witten diagram. We then assess its contribution to the differential cross section, including the t-channel contribution from the graviton or tensor glueball exchange. In Sec. IV we give a numerical assessment of each contribution to the differential and total cross sections in the threshold region. Our conclusions are in Sec. V.

\section{TRANSITION FORM FACTORS}

The transition form factors for pentaquark production $\gamma+$ $p \rightarrow X$ and decay $X \rightarrow V+p$, were derived using the holographic construction in [10] to which we refer for completeness. In short, the transition form factor is given by the 3-point Witten diagram illustrated in Fig. 1. It is composed of the U(1) gauge field in bulk, coupled with a Dirac fermion (probed by the nucleon at the boundary) and an additional Dirac fermion probed by a pentaquark in the $\left[\frac{1}{2} \frac{1}{2}^{-}\right]$representation. The coupling to a pentaquark in the $\left[\frac{1}{2} \frac{3}{2}^{-}\right]$representation will follow by symmetry below.

\section{A. Sakai-Sugimoto model}

In the Sakai-Sugimoto model [34] the holographic pentaquarks are realized as topological bound states of a core instanton with a degenerate doublet of heavy-light mesons $\left[D, D^{*}\right]$ or $\left[B, B^{*}\right]$ that transmute to spin- $\frac{1}{2}$ fermions [3]. Their decay widths were recently analyzed in [10]. The masses and

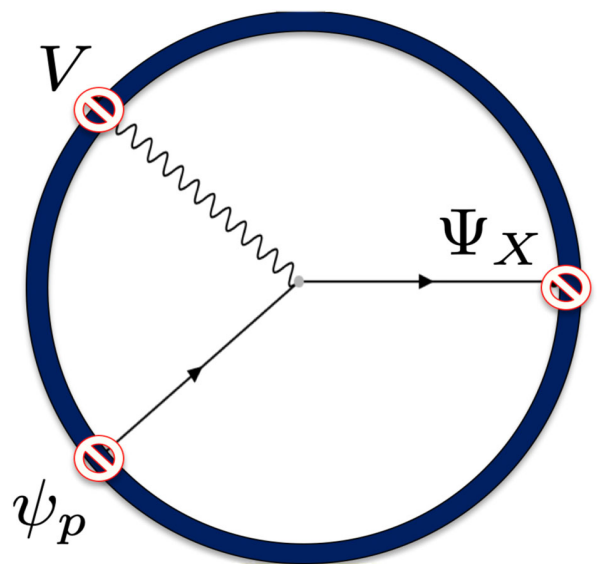

FIG. 1. Witten diagram for the U(1) transition vertex $V+\psi_{p} \rightarrow \Psi_{X}$.

total widths of the three charm pentaquark states are fixed to those reported empirically by $\mathrm{LHCb}[1]$

$m_{P_{c}}=4311.9 \pm 0.7 \mathrm{MeV} \quad \Gamma_{P_{c}}=9.8 \pm 2.7 \mathrm{MeV}$

$m_{P_{c}}=4440.3 \pm 1.3 \mathrm{MeV} \quad \Gamma_{P_{c}}=20.6 \pm 4.9 \mathrm{MeV}$

$m_{P_{c}}=4457.3 \pm 0.6 \mathrm{MeV} \quad \Gamma_{P_{c}}=6.4 \pm 2.0 \mathrm{MeV}$.

For the unobserved bottom pentaquarks, the theoretical masses and widths estimates from the more recent holographic analyses $[9,10]$

$\begin{array}{ll}m_{P_{b}}=11155 \mathrm{MeV} & \Gamma_{P_{b}}=17.87 \Gamma=71 \pm 18 \mathrm{MeV} \\ m_{P_{b}}=11163 \mathrm{MeV} & \Gamma_{P_{b}}=10.76 \Gamma=43 \pm 11 \mathrm{MeV} \\ m_{P_{b}}=11167 \mathrm{MeV} & \Gamma_{P_{b}}=8.15 \Gamma=33 \pm 8 \mathrm{MeV}\end{array}$

with $\Gamma=4 \pm 1 \mathrm{MeV}$ fixed empirically. The holographic transition couplings will be detailed below. Note that in [3] both charm and bottom heavy-light exotics were described using a single heavy brane. Double heavy branes are in general needed for exotics with mixed charm and bottom. However, this is not needed for the process to follow, to the order quoted in the heavy quark mass.

In the heavy quark limit, the U(1) transition form factor $X=P_{c, b} \rightarrow V+p$ was obtained in the form [10]

$\langle P|\vec{J}(\vec{x}-\vec{X})| X\rangle=G(\vec{P})\left(i \vec{P} \times \bar{v}_{\bar{Q}} \vec{\sigma} u_{Q}\right)(2 \pi)^{3} \delta^{3}\left(P^{\prime}-P\right)$,

with

$$
\begin{aligned}
G(\vec{P})= & \lambda \sqrt{\frac{m_{N}}{m_{X}}} \sum_{n=\text { odd }} \int d Z\left(\Psi_{10}(Z) \frac{\varphi_{n}(Z)}{\sqrt{\kappa}} \Psi_{10}(Z)\right) \\
& \times \frac{g_{n}}{E^{2}-\vec{P}^{2}-\tilde{m}_{n}^{2}},
\end{aligned}
$$


where $\kappa$ is the flavor brane tension. The modular wave functions $\Psi_{l n_{\rho}}(Z)$ for the nucleon and the pentaquark states are similar in the ground state

$$
\Psi_{10}(Z)=\left(\frac{2 M_{0}}{\sqrt{6} \pi}\right)^{\frac{1}{4}} e^{-\frac{M_{0}}{\sqrt{6}} Z^{2}}
$$

with $M_{0}=8 \pi^{2} \kappa$ the bulk instanton bare mass in units of $M_{K K}$. We recall that in the bound state approach, the negative intrinsic parity of the pentaquark is due to the attached D-meson (B-meson) multiplet to the instanton core. This is manifest in the matrix element $\bar{u}_{Q} \vec{\sigma} v_{\bar{Q}}$ in (3) after spin-statistics transmutation [3]. The attachment is BPS in leading order. The modular wave function (5) is parity even, while the emerging attachment $Q \bar{Q}$ is parity odd.

The sum in (4) is over the tower of $1^{--}$vector mesons in bulk. In other words, the transition form factor (4) is vectorlike. It is sourced by a bound pentaquark composed of an instanton core made of light flavors, with an emerging heavy $Q \bar{Q}$ attachment through transmutation. The $1^{--}$ vectors satisfy the eigenvalue equation [34]

$$
-h^{-1}(Z) \partial_{Z}\left(k(Z) \partial_{Z} \varphi_{n}(Z)\right)=\lambda_{n} \varphi_{n}(Z),
$$

with warping functions $h(Z), k(Z)$ [34]. The eigenvalues are related to the masses of the vector mesons $\tilde{m}_{n}=\lambda_{n}$ in units of $M_{K K}$, which do not Reggeize. The vector meson decay constants are $g_{n} \sim 2 \kappa\left(Z \varphi_{2 n-1}(Z)\right)$ at asymptotic $Z$. The eigenvalues are normalized so that $\varphi_{n}(Z) \sim 1 / \sqrt{\kappa}$ with $\kappa$ the brane tension parameter, and their parity is $\varphi_{n}(-Z)=$ $(-1)^{n+1} \varphi_{n}(Z)$ according to the mode number. The $1^{--}$ trajectory carries odd-n, with the lowest rho-meson mass $m_{1}=0.67$.

The boundary gauge invariant, relativistic and local coupling between the nucleon and the pentaquark corresponding to (3)-(4) is

$$
\frac{e \alpha_{X}}{m_{X}} \bar{\Psi}(x) \gamma_{\mu \nu} F^{\mu \nu}(x) \psi(x),
$$

with matching positive parity between the bulk modular wave function $\Psi_{10}(Z)$ in (5) and the boundary field $\Psi(x)$ with spin-isospin assignment $\left[\frac{1}{2} \frac{1}{2}^{+}\right]$. In the Appendix, we show that (7) reproduces the pentaquark decay width $X \rightarrow$ $V+p$ following from (3)-(4) as recently derived in the Sakai-Sugimoto model for heavy baryons [10]. It is Paulilike with a transition magnetic moment

$$
\frac{\mu_{X}}{\mu_{N}}=\frac{e \alpha_{X}}{\mu_{N} m_{X}} \rightarrow 4 \eta_{X} \frac{m_{N}^{2}}{m_{\rho}^{2}}
$$

in nuclear Bohr magneton $\mu_{N}=e / 2 m_{N}$. The last identity follows from mapping to the soft wall, and using (34) below. For a charm pentaquark mass $m_{X} \sim 4440 \mathrm{MeV}$ we have $\mu_{4440} / \mu_{N} \sim 0.78$, with the transition coupling $\left|\eta_{4440}\right|=$ 0.134 using (40). The transition magnetic moment is smaller for bottom pentaquarks since $\mu_{P_{b}} / \mu_{P_{c}}=\left(m_{P_{c}} / m_{P_{b}}\right)^{\frac{3}{2}}$ following from (8) and (A5).

\section{B. Soft wall model}

In the Sakai-Sugimoto model the resummed $1^{--}$spectrum does not Reggeize, as we noted earlier. As a result, the ensuing U(1) form factors do not obey the hard scattering rules asymptotically. To fix this we will use instead the soft wall model which Reggeizes, while maintaining the same Pauli-like form factors for all holographic pentaquark states in the heavy quark limit by matching.

We note that the topological construction used in $[9,10]$ can be revisited in the soft wall approach as in [35], but this would require a new and more extensive study. Rather, we will follow the Reggeized approach advocated in [36] for the electromagnetic form factors, where the unknown effective Pauli coupling of the nucleon to the electromagnetic current in a warped 5-dimensional space, is extracted from the 10-dimensional Sakai-Sugimoto construction.

With this in mind, the bulk interaction vertex between the Dirac fermion $\psi$ and the pentaquark field $\Psi$ is

$\eta_{X} \int d z d^{4} x \sqrt{|g(z)|} e^{-\phi_{N}(z)} \sum_{\xi=1,2} \bar{\Psi}_{\xi} e_{A}^{M} e_{B}^{N} \sigma^{A B} F_{M N} \psi_{\xi}$,

with $g_{M N}(z)=\left(R^{2} / z^{2}\right)(+,-,-,-,-)$ the mostly negative $\mathrm{AdS}_{5}$ metric, $e_{A}^{M}$ its associated vierbeins, $\sigma^{A B}=\frac{i}{2}\left[\Gamma^{A}, \Gamma^{B}\right]$, and $\phi_{N}(z)=\tilde{\kappa}_{N}^{2} z^{2}$ the dilaton in the bulk filling baryonic brane in the soft wall approximation. Note that the "pentaquark" bulk field $\Psi(x, z)$ is the analogue of the modular field (5) with positive parity. This is important in describing the analogue of the vector form factor (4) in the soft wall construction with manifest Regge behavior. The labels $\xi=1,2$ refer to the chiralities of the fermionic operators inserted at the boundary [37] and

$$
\frac{\eta_{X}}{\tilde{\kappa}_{N}}=\frac{\alpha_{X}}{m_{X}}
$$

is fixed by comparing the two holographic constructions. The initial and final state wave functions for the nucleon (pentaquark) states will be defined as

$$
\begin{aligned}
\psi(z) & =\psi_{L}(z)+\psi_{R}(z) \\
\psi_{L, R}(z) & =e^{-i P \cdot x} \frac{1}{2}\left(1 \mp \gamma^{5}\right) u_{s}(P) \frac{z^{2}}{R^{2}} \tilde{f}_{L, R}(z),
\end{aligned}
$$

where the left and right modes satisfy the equation of motion 


$$
\begin{aligned}
& {\left[-\partial_{z}^{2}+\tilde{\kappa}_{N}^{2} z^{2}+2 \tilde{\kappa}_{N}^{2}\left(M_{N} \mp \frac{1}{2}\right)+\frac{M_{N}\left(M_{N} \pm 1\right)}{z^{2}}\right] \tilde{f}_{L, R}(z)} \\
& =P^{2} \tilde{f}_{L, R}(z) .
\end{aligned}
$$

with $M_{N}=\Delta_{N}-2=\tau_{N}-\frac{3}{2}$ the anomalous dimension of the nucleon (pentaquark) source with twist $\tau_{N}$. The mass spectrum Reggeizes radially

$$
P^{2}=m_{n}^{2}=4 \tilde{\kappa}_{N}^{2}\left(n+M_{N}+\frac{1}{2}\right) .
$$

with the normalized states

$$
\begin{aligned}
& \tilde{f}_{L}^{n}(z)=\sqrt{\frac{2 \Gamma(n+1)}{\Gamma\left(n+M_{N}+\frac{3}{2}\right)}} \tilde{\kappa}_{N}^{M_{N}+\frac{3}{2}} z^{M_{N}+1} L_{n}^{M_{N}+\frac{1}{2}}\left(\tilde{\kappa}_{N}^{2} z^{2}\right) \\
& \equiv C_{L}\left(M_{N}, n\right) \kappa^{M_{N}+\frac{3}{2}} z^{M_{N}+1} L_{n}^{M_{N}+\frac{1}{2}}\left(\tilde{\kappa}_{N}^{2} z^{2}\right) \\
& \tilde{f}_{R}^{n}(z)=\sqrt{\frac{2 \Gamma(n+1)}{\Gamma\left(n+M_{N}+\frac{1}{2}\right)}} \tilde{\kappa}_{N}^{M_{N}+\frac{1}{2}} z^{M_{N}} L_{n}^{M_{N}-\frac{1}{2}}\left(\tilde{\kappa}_{N}^{2} z^{2}\right) \\
& \equiv C_{R}\left(M_{N}, n\right) \tilde{\kappa}_{N}^{M_{N}+\frac{1}{2}} z^{M_{N}} L_{n}^{M_{N}-\frac{1}{2}}\left(\tilde{\kappa}_{N}^{2} z^{2}\right) .
\end{aligned}
$$

For the proton, one has $n=0$ and the anomalous dimension is $M_{N} \equiv \tau_{N}-\frac{3}{2}$. For pentaquark states, one can choose a generic anomalous dimension $M_{X}$ and radial quantum number $n=n_{X}$, assuming a universal baryonic slope $\tilde{\kappa}_{X}=\tilde{\kappa}_{N}$. The twist $\tau_{X}$ and the anomalous dimensions $M_{X}$ are fixed below. Note that for ground state $(n=0)$ the bulk fermionic modes simplify

$$
\begin{aligned}
\psi_{L}\left(z, \tau_{N}\right) & =z^{\Delta_{N}} \times \tilde{\psi}_{L}\left(z, \tau_{N}\right) \\
& =n_{L}\left(\tilde{\kappa}_{N}, \tau_{N}\right) \times \tilde{\kappa}_{N}^{2\left(\tau_{N}-1\right)} z^{2\left(\tau_{N}-1\right)} \times z^{\Delta_{N}}, \\
\psi_{R}\left(z, \tau_{N}\right) & =z^{\Delta_{N}} \times \tilde{\psi}_{R}\left(z, \tau_{N}\right) \\
& =n_{R}\left(\tilde{\kappa}_{N}, \tau_{N}\right) \times \tilde{\kappa}_{N}^{2\left(\tau_{N}-1\right)-1} z^{2\left(\tau_{N}-1\right)-1} \times z^{\Delta_{N}},
\end{aligned}
$$

with the normalization factors

$$
\begin{aligned}
& n_{L}\left(\tilde{\kappa}_{N}, \tau_{N}\right)=\frac{1}{\tilde{\kappa}_{N}^{\tau-2}} \times \sqrt{\frac{2}{\Gamma\left(\tau_{N}\right)}}, \\
& n_{R}\left(\tilde{\kappa}_{N}, \tau_{N}\right)=n_{L}\left(\tilde{\kappa}_{N}, \tau\right) \times \sqrt{\tau_{N}-1} .
\end{aligned}
$$

\section{Transition form factor: $\gamma+\boldsymbol{p} \rightarrow \boldsymbol{P}_{c}$}

The re-summed U(1) bulk-to-boundary propagator in the soft wall model is [37,38],

$$
\begin{aligned}
\mathcal{V}\left(Q, z, \tilde{\kappa}_{V}\right) & =\tilde{\kappa}_{V}^{2} z^{2} \Gamma\left(1+a_{Q}\right) \mathcal{U}\left(1+a_{Q} ; 2 ; \tilde{\kappa}_{V}^{2} z^{2}\right) \\
& =\tilde{\kappa}_{V}^{2} z^{2} \int_{0}^{1} \frac{d x}{(1-x)^{2}} x^{a_{Q}} \exp \left(-\tilde{\kappa}_{V}^{2} z^{2} \frac{x}{1-x}\right) \\
& =a_{Q} \int_{0}^{1} d x x^{a_{Q}-1} \exp \left(-\tilde{\kappa}_{V}^{2} z^{2} \frac{x}{1-x}\right),
\end{aligned}
$$

with $\mathcal{U}$ the Kummer function and $a_{Q}=Q^{2} /\left(4 \tilde{\kappa}_{V}^{2}\right)$. We note the normalizations $\mathcal{V}\left(0, z, \tilde{\kappa}_{V}\right)=\mathcal{V}\left(Q, 0, \tilde{\kappa}_{V}\right)=1$. As a result, for the Pauli-like coupling (9), the form factor becomes

$$
\begin{gathered}
\mathcal{W}\left(Q^{2}\right)=\frac{\eta_{X}}{\tilde{\kappa}_{N}} \bar{u}\left(P^{\prime}\right) i(\not \phi \mid-\not \phi) u(P) \mathcal{I}\left(n_{X}, Q^{2}\right), \\
\mathcal{I}\left(n_{X}, Q^{2}\right)=\frac{1}{2}\left(\mathcal{I}_{L R}\left(n_{X}, Q^{2}\right)+\mathcal{I}_{R L}\left(n_{X}, Q^{2}\right)\right),
\end{gathered}
$$

where the scalar form factors $\mathcal{I}_{L R}$ and $\mathcal{I}_{R L}$ read

$$
\begin{aligned}
\mathcal{I}_{L R}\left(n_{X}, Q^{2}\right)= & \frac{1}{2} C_{L}\left(M_{X}, n_{X}\right) C_{R}\left(M_{N}, 0\right) \\
& \times \int_{0}^{\infty} d w w^{\frac{M_{X}+M_{N}+3}{2}} e^{-w} L_{n_{X}}^{M_{X}+\frac{1}{2}}(w) \mathcal{U} \\
& \times\left(1+a_{Q} ; 2, w\right) \Gamma\left(1+a_{Q}\right), \\
\mathcal{I}_{R L}\left(n_{X}, Q^{2}\right)= & \frac{1}{2} C_{R}\left(M_{X}, n_{X}\right) C_{L}\left(M_{N}, 0\right) \\
& \times \int_{0}^{\infty} d w w^{\frac{M_{X}+M_{N}+3}{2}} e^{-w} L_{n_{X}}^{M_{X}-\frac{1}{2}}(w) \mathcal{U} \\
& \times\left(1+a_{Q} ; 2, w\right) \Gamma\left(1+a_{Q}\right) .
\end{aligned}
$$

Notice that for identical anomalous dimensions $M_{X}=M_{N}$, the above integrals can be analytically evaluated.

\section{Transition form factor: $\boldsymbol{P}_{\boldsymbol{c}} \rightarrow \boldsymbol{V}+\boldsymbol{p}$}

When the $U(1)_{V}$ probing source goes on-shell a vector meson $V=J / \Psi, \Upsilon$ is produced. Using the LSZ reduction, we first note that

$w \mathcal{U}\left(1+a_{Q} ; 2, w\right) \Gamma\left(1+a_{Q}\right)=\sum_{n} \frac{\phi_{n}(z) F_{n}}{Q^{2}+\tilde{m}_{n}^{2}} \rightarrow \phi_{n}(z)$,

with the Reggeized meson spectrum $\tilde{m}_{n}^{2}=2 \tilde{\kappa}_{V}^{2}\left(n+1+C_{V}\right)$ shifted by $C_{V}=M_{V}^{2} / 2 \tilde{\kappa}^{2}-1$ and

$$
\phi_{n}(z)=\sqrt{\frac{2}{n+1}} \tilde{\kappa}_{V}^{2} z^{2} L_{n}^{1}\left(\tilde{\kappa}_{V}^{2} z^{2}\right),
$$

the normalized wave functions for vector mesons $V$ in bulk. Therefore, the form factor for $X \rightarrow V+p$ is 


$$
\begin{aligned}
\mathcal{W}\left(-m_{n}^{2}\right) & =\frac{\eta_{X}}{\tilde{\kappa}_{N}} \bar{u}\left(P^{\prime}\right) i(\phi \not \mid-\not \phi \phi) u(P) \mathcal{I}\left(n_{X},-m_{n}^{2}\right), \\
\mathcal{I}\left(n_{X},-m_{n}^{2}\right) & =\frac{1}{2}\left(\mathcal{I}_{L R}\left(n_{X},-m_{n}^{2}\right)+\mathcal{I}_{R L}\left(n_{X},-m_{n}^{2}\right)\right),
\end{aligned}
$$

with

$$
\begin{aligned}
& \mathcal{I}_{L R}\left(n_{X},-m_{n}^{2}\right)=\frac{1}{2} C_{L}\left(M_{X}, n_{X}\right) C_{R}\left(M_{N}, 0\right) \sqrt{\frac{2}{n+1}} \int_{0}^{\infty} d w w^{\frac{M_{X}+M_{N}+3}{2}} e^{-w} L_{n_{X}}^{M_{X}+\frac{1}{2}}(w) L_{n}^{1}(w), \\
& \mathcal{I}_{R L}\left(n_{X},-m_{n}^{2}\right)=\frac{1}{2} C_{R}\left(M_{X}, n_{X}\right) C_{L}\left(M_{N}, 0\right) \sqrt{\frac{2}{n+1}} \int_{0}^{\infty} d w w^{\frac{M_{X}+M_{N}+3}{2}} e^{-w} L_{n_{X}-\frac{1}{2}}^{M}(w) L_{n}^{1}(w) .
\end{aligned}
$$

\section{DIFFERENTIAL CROSS SECTION}

In holography, the photoproduction of charmonium (bottomonium) near threshold receives s- and t-channel contributions. The holographic t-channel contribution in Fig. 2 (right) is known [38,39]. It is dominated by the graviton exchange (tensor glueball) near threshold as detailed in [38]

$$
\left(\frac{d \sigma}{d t}\right)_{G}=\frac{\mathcal{N}^{2} e^{2}}{64 \pi\left(s-m_{N}^{2}\right)^{2}} \times \frac{A^{2}(Q)}{A^{2}(0)}\left(2-\frac{t}{2 m_{N}^{2}}\right) F(s, t)
$$

with the gravitational form factor

$$
\begin{aligned}
A(Q)= & A(0)\left(a_{Q}+1\right)\left(-\left(1+a_{Q}+2 a_{Q}^{2}\right)\right. \\
& \left.+2\left(a_{Q}+2 a_{Q}^{3}\right) \Phi\left(-1,1, a_{Q}\right)\right),
\end{aligned}
$$

$\Phi\left(-1,1, a_{Q}\right)$ refers to the Lerch $\Phi$ function, with $a_{Q}=$ $Q^{2} / 8 \tilde{\kappa}_{V}^{2}$ and $Q^{2}=-t$. It is remarkably dipole-like. The extra kinematical factor $F(s, t)$ can be found in [38].
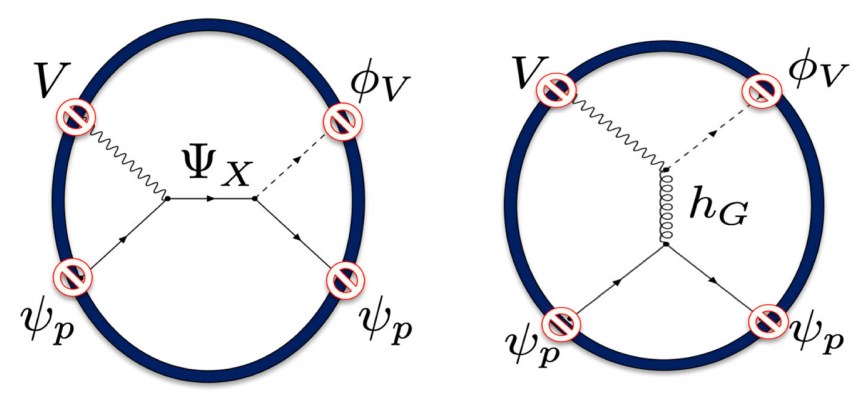

FIG. 2. Witten diagram for the photoproduction process $V+$ $p \rightarrow p+V$ of a vector meson $V$ off a proton $\psi_{p}$. The pentaquark $\Psi_{X}$ excitation in the s-channel with no crossing shown (left) is added to the graviton exchange $h_{G}$ in the t-channel (right).
It scales like $s^{4}$ at large $s$ but otherwise varies weakly with $t$ near treshold.

\section{A. Spin $\frac{1}{2}$}

The s-channel contribution is new, and will be restricted to the possible photoexcitation of a pentaquark as a possible threshold enhancement. More specifically, the s-channel $\left[\frac{1}{2} \frac{1}{2}^{-}\right]_{S=0,1}$ pentaquark contribution in Fig. 2 (left) plus crossing give

$$
\begin{aligned}
& \mathcal{M}_{\frac{1}{2}}=\sum_{n_{X}} \frac{\left[\frac{\eta_{X}}{\tilde{\kappa}_{N}} \mathcal{I}(0,0)\right]\left[{\underline{\eta_{X}}}_{\tilde{\kappa}_{N}} \mathcal{I}\left(0,-m_{V}^{2}\right)\right]}{s-m_{n_{X}}^{2}+i m_{n_{X}} \Gamma_{n_{X}}} \\
& \times \bar{u}\left(p^{\prime}, S^{\prime}\right)\left(\not k^{\prime} \phi^{\star}-\not \phi^{\star} \not k^{\prime}\right)\left(\not p+m_{n_{X}}\right) \\
& \times(\phi \not k-\not k \phi) u(p, S)+\mathrm{cross}
\end{aligned}
$$

Keeping only the ground states $n_{X}=0$ contribution, then we approximately have

$\mathcal{M}_{\frac{1}{2}} \approx e^{2} \frac{\left[\frac{\eta_{X}}{\tilde{\kappa}_{N}} \mathcal{I}(0,0)\right]\left[\frac{\eta_{X}}{\tilde{\kappa}_{N}} \mathcal{I}\left(0,-m_{V}^{2}\right)\right]}{s-m_{X}^{2}+i m_{X} \Gamma_{X}} \mathcal{A}_{\frac{1}{2}}\left(S^{\prime}, S\right)+$ cross,

with $\eta_{X}=\alpha_{X} / m_{X}, m_{X}=m_{n_{X}=0}$, and $\Gamma_{X}=\Gamma_{n_{X}=0}$ and

$$
\begin{aligned}
\mathcal{A}_{\frac{1}{2}}\left(S^{\prime}, S\right)= & \bar{u}\left(p^{\prime}, S^{\prime}\right)\left(\not \not^{\prime} \phi^{\star}-\phi^{\star} \not k^{\prime}\right)\left(p A k+m_{n_{X}}\right) \\
& \times(\phi \not k-\not k \phi) u(p, S)
\end{aligned}
$$

is the generic-spin structure for the Pauli-exchange. For the near-threshold production, $p \approx \frac{m_{X}}{2}(1,1,0,0)$ and $k \approx$ $\frac{m_{X}}{2}(1,-1,0,0)$ are close to the light-cone, while $\epsilon$ and $\epsilon^{\prime}$ are transversally polarized. The crossing contribution is expected to be small for a far off-shell $X=P_{c, b}$. It will be ignored. Hence 


$$
\begin{aligned}
\frac{1}{4} \sum_{S, S^{\prime}}\left|\mathcal{A}_{\frac{1}{2}}\left(S^{\prime}, S\right)\right|^{2}= & 4 \operatorname{Tr}\left[( \not p + \not k + m _ { X } ) \left(3 m_{N} k^{2}\right.\right. \\
& \left.+k^{2} \not \not-4 k \cdot p \not k\right)\left(\not p+\not k+m_{X}\right) \\
& \times\left(3 m_{N} k^{\prime 2}+\left(k^{\prime 2} \not p^{\prime}-4 k^{\prime} \cdot p^{\prime} \not \not^{\prime}\right)\right]
\end{aligned}
$$

after summing over the polarizations and spins. Here we have used the identity

$$
\begin{aligned}
& \left(\gamma^{\mu} \not k-\not k \gamma^{\mu}\right)\left(\not p+m_{N}\right)\left(\not k \gamma_{\mu}-\gamma_{\mu} \not k\right) \\
& =12 k^{2} m_{N}+4 k^{2} \not p-16 k \cdot p \not k .
\end{aligned}
$$

In general, the trace can be evaluated using $k^{2}=-Q^{2}$ for the electro-production process, and the standard relation $s+t+u=2 m_{N}^{2}+m_{X}^{2}-Q^{2}$

$$
\begin{aligned}
\mathcal{A}_{\frac{1}{2}}\left(s, t ; Q^{2}\right) \equiv & \frac{1}{4} \sum_{S, S^{\prime}}\left|\mathcal{A}_{\frac{1}{2}}\left(S^{\prime}, S\right)\right|^{2} \\
= & 8\left(-4 m_{N}^{6}\left[3 m_{X}^{2}-3 Q^{2}+4 s+t\right]+m_{N}^{4}\left[m_{X}^{2}\left(-37 Q^{2}+20 s-2 t\right)+Q^{2}(2 t-20 s)+12 s(2 s+t)\right]\right. \\
& +m_{N}^{2}\left[m_{X}^{2}\left(Q^{2}(t-22 s)+9 Q^{4}+4 s(t-s)\right)-9 m_{X}^{4} Q^{2}-4 s\left(Q^{2}(t-s)+s(4 s+3 t)\right)\right] \\
& \left.+4 m_{N}^{8}-m_{X}^{2} s\left[Q^{2}(5 s+t)+Q^{4}+2 s(2 s+t)\right]+m_{X}^{4} Q^{2}\left[Q^{2}+s\right]+2 s^{2}\left[Q^{2}(2 s+t)+2 s(s+t)\right]\right) .
\end{aligned}
$$

The s-channel contribution of $\left[\frac{1}{2} \frac{1}{2}^{-}\right]_{S=0,1}$ pentaquark state to the differential cross section for photoproduction is

$$
\left(\frac{d \sigma}{d t}\right)_{X} \approx \frac{e^{2}}{16 \pi\left(s-m_{N}^{2}\right)^{2}}\left|\frac{\left[\frac{\eta_{X}}{\tilde{\kappa}_{N}} \mathcal{I}\left(0,-Q^{2}\right)\right]\left[\frac{\eta_{X}}{\tilde{\kappa}_{N}} \mathcal{I}\left(0,-m_{V}^{2}\right)\right]}{s-m_{X}^{2}+i m_{X} \Gamma_{X}}\right|^{2} \mathcal{A}_{\frac{1}{2}}(s, t ; 0)
$$

The kinematic factor $\mathcal{A}(s, t ; 0)$ is given by Eq. (31). The vertex form factors are explicitly

$$
\begin{aligned}
\frac{\eta_{X}}{\tilde{\kappa}_{N}} \times \mathcal{I}\left(0,-Q^{2}\right) & =\frac{\eta_{X}}{\tilde{\kappa}_{N}} \times \tilde{\kappa}_{N} \times \int d z e^{-\tilde{\kappa}_{N}^{2} z^{2}} \frac{\mathcal{V}\left(Q, z, \tilde{\kappa}_{V}\right)}{2 z^{\tau_{N}+\tau_{X}-4}}\left[\tilde{\psi}_{L}\left(z, \tau_{X}\right) \tilde{\psi}_{R}\left(z, \tau_{N}\right)+\tilde{\psi}_{R}\left(z, \tau_{X}\right) \tilde{\psi}_{L}\left(z, \tau_{N}\right)\right] \\
& =\frac{\eta_{X}}{\tilde{\kappa}_{N}} \times \frac{\Gamma\left(a_{Q}+1\right)\left(\sqrt{\tau_{N}-1}+\sqrt{\tau_{X}-1}\right) \Gamma\left(\frac{1}{2}\left(\tau_{N}+\tau_{X}\right)\right) \Gamma\left(\frac{1}{2}\left(\tau_{N}+\tau_{X}+2\right)\right)}{4 \Gamma\left(a_{Q}+\tau_{X}+1\right) \sqrt{\Gamma\left(\tau_{N}\right) \Gamma\left(\tau_{X}\right)}}, \\
\frac{\eta_{X}}{\tilde{\kappa}_{N}} \times \mathcal{I}\left(0,-m_{V}^{2}\right) & =\frac{\eta_{X}}{\tilde{\kappa}_{N}} \times \tilde{\kappa}_{N} \times \int d z e^{-\tilde{\kappa}_{N}^{2} z^{2}} \frac{\sqrt{2} \tilde{\kappa}_{V}^{2} z^{2}}{2 z^{\tau_{N}+\tau_{X}-4}}\left[\tilde{\psi}_{L}\left(z, \tau_{X}\right) \tilde{\psi}_{R}\left(z, \tau_{N}\right)+\tilde{\psi}_{R}\left(z, \tau_{X}\right) \tilde{\psi}_{L}\left(z, \tau_{N}\right)\right] \\
& =\frac{\eta_{X}}{\tilde{\kappa}_{N}} \times \frac{\tilde{\kappa}_{V}^{2}}{\tilde{\kappa}_{N}^{2}} \times \frac{\left(\sqrt{\tau_{N}-1}+\sqrt{\tau_{X}-1}\right) \Gamma\left(\frac{1}{2}\left(\tau_{N}+\tau_{X}+2\right)\right)}{2 \sqrt{2} \sqrt{\Gamma\left(\tau_{N}\right) \Gamma\left(\tau_{X}\right)}},
\end{aligned}
$$

with $a_{Q}=Q^{2} / 4 \tilde{\kappa}_{V}^{2}$, after using (17) and (15) respectively. We fix the Regge slopes and anomalous dimensions as follows

$$
\begin{aligned}
4 \tilde{\kappa}_{N}^{2} & =4 \tilde{\kappa}_{\rho}^{2}=m_{\rho}^{2}, \quad \tau_{N}=1+\frac{m_{N}^{2}}{m_{\rho}^{2}}=2.465, \\
\tau_{X} & =1+\frac{m_{X}^{2}}{m_{\rho}^{2}} .
\end{aligned}
$$

and set $\tilde{\kappa}_{V}=\tilde{\kappa}_{J / \Psi}=1.03784 \mathrm{GeV}$ from the high-energy electroproduction data for $J / \Psi$ as was done in [38,39].

\section{B. Pauli coupling constant}

In so far, the only unknown parameter in our set-up (for the soft wall holographic QCD model) is the Pauli coupling
$\eta_{X}$ which, in principle, can be fixed by the experimental value of the branching ratio for $\operatorname{Br}(X \rightarrow V+p)$ which is lacking. Therefore, we use the theoretically computed $\operatorname{Br}(X \rightarrow V+p)$ from the Sakai-Sugimoto holographic QCD model in [10] to fix $\eta_{X}$.

In the soft wall, the partial decay width for $X\left(p_{X}\right) \rightarrow$ $V\left(k^{\prime}\right)+p\left(p^{\prime}\right)$ is given by

$$
\begin{aligned}
\frac{d \Gamma_{X V}}{d \Omega}= & \frac{\left|\vec{k}_{V}\right|}{32 \pi^{2} m_{X}^{2}} \times \frac{1}{2} \sum_{\text {pol }} \frac{1}{2} \sum_{\text {spin }}\left|\mathcal{A}_{X \rightarrow J / \Psi p}\left(m_{X}, m_{N}, m_{V}\right)\right|^{2} \\
= & \frac{\left|\vec{k}_{V}\right|}{32 \pi^{2} m_{X}^{2}} \times\left[\frac{\eta_{X}}{\tilde{\kappa}_{N}} \mathcal{I}\left(0,-m_{V}^{2}\right)\right]^{2} \\
& \times \mathcal{A}_{\Gamma_{X V}}\left(m_{X}, m_{N}, m_{V}\right)
\end{aligned}
$$


with $\vec{k}^{\prime}=\vec{k}_{V}$, the 3-momentum of the emitted vector meson, given by

$$
\left|\vec{k}_{V}\right|=\frac{1}{2 m_{X}} \sqrt{m_{X}^{4}-2\left(m_{V}^{2}+m_{N}^{2}\right) m_{X}^{2}+\left(m_{V}^{2}-m_{N}^{2}\right)^{2}},
$$

and we have defined

$$
\begin{aligned}
\mathcal{A}_{\Gamma_{X V}}\left(m_{X}, m_{N}, m_{V}\right)= & \frac{1}{2} \sum_{\mathrm{pol}} \frac{1}{2} \operatorname{Tr}\left[\left(\not p^{\prime}+m_{N}\right)\left(\phi^{\prime \prime} \not k^{\prime}-\not k^{\prime} \phi^{\prime \prime}\right)\left(\not \not{ }_{X}+m_{X}\right)\left(\not k^{\prime} \phi^{\prime *}-\not \phi^{\prime *} \not k^{\prime}\right)\right] \\
= & -12 m_{V}^{2} m_{N} m_{X}-4 m_{V}^{2} p \cdot p^{\prime}+16 k^{\prime} \cdot p k^{\prime} \cdot p^{\prime} \\
= & -12 m_{V}^{2} m_{N} m_{X}-2 m_{V}^{2}\left(m_{N}^{2}+m_{X}^{2}-m_{V}^{2}\right) \\
& +4\left(m_{X}^{2}-m_{V}^{2}-m_{N}^{2}\right)\left(m_{X}^{2}+m_{V}^{2}-m_{N}^{2}\right),
\end{aligned}
$$

where we have used

$$
\sum_{s=1,2,3} \epsilon_{s}^{\prime \mu} \epsilon_{s}^{\prime * \nu}=-\eta^{\mu \nu}+\frac{k^{\prime \mu} k^{\prime \nu}}{m_{V}^{2}} .
$$

Therefore, we have

$$
\begin{aligned}
\Gamma_{X V}= & \frac{\left|\vec{k}_{V}\right|}{8 \pi m_{X}^{2}} \times\left[\frac{\eta_{X}}{\tilde{\kappa}_{N}} \mathcal{I}\left(0,-m_{V}^{2}\right)\right]^{2} \\
& \times \mathcal{A}_{\Gamma_{X V}}\left(m_{X}, m_{N}, m_{V}\right) .
\end{aligned}
$$

which allows to fix the Pauli coupling $\eta_{X}$ given the partial widths $\Gamma_{X V}$

$$
\eta_{X}^{2}=\frac{\Gamma_{X V}}{\frac{\left|\vec{k}_{V}\right|}{8 \pi m_{X}^{2}} \times\left[\frac{1}{\tilde{\kappa}_{N}} \mathcal{I}\left(0,-m_{V}^{2}\right)\right]^{2} \times \mathcal{A}_{\Gamma_{X V}}\left(m_{X}, m_{N}, m_{V}\right)} .
$$

Note that the photodecay widths $\Gamma_{X \gamma}$ of the holographic pentaquarks in the process $X\left(p_{X}\right) \rightarrow \gamma(k)+p(p)$ are fixed by similar arguments

$$
\begin{aligned}
\Gamma_{X \gamma}= & \frac{|\vec{k}|}{8 \pi m_{X}^{2}} \times\left[\frac{\eta_{X}}{\tilde{\kappa}_{N}} \mathcal{I}\left(0,-Q^{2}\right)\right]^{2} \\
& \times \mathcal{A}_{\Gamma_{X_{Y}}}\left(m_{X}, m_{N}, m_{V} \rightarrow \sqrt{-Q^{2}}\right) .
\end{aligned}
$$

The partial widths $\Gamma_{X V}$ were evaluated in the holographic construction in [10]. More specifically: For $X=P_{c}(4440)$ and $V=J / \Psi$, using $\Gamma_{X V}=0.00034 \pm 0.00009 \mathrm{GeV}$, we find its Pauli coupling to be $\eta_{X}(4440)=0.134 \pm 0.017$. For this decay process, the branching ratio is $\mathrm{Br}_{X}=$ $\Gamma_{X V} / \Gamma_{X}=1.8 \%$, which is close to the upper bound $\mathrm{Br}_{X, \text { GlueX }}<2 \%$ set by GlueX [40], and the lower bound $\mathrm{Br}_{X}>0.05 \%$ empirically argued in [41]. For $X=P_{c}$ (4312) and $V=J / \Psi$, using $\Gamma_{X V}=0.000056 \pm 0.000014 \mathrm{GeV}$, we find its Pauli coupling to be $\eta_{X}(4312)=0.044 \pm 0.005$, and the branching ratio is $\mathrm{Br}_{X}=0.3 \%$ within the bounds.
For $X=P_{c}(4457)$ and $V=J / \Psi$, using $\Gamma_{X V}=0.00017 \pm$ $0.000043 \mathrm{GeV}$, we find its Pauli coupling to be $\eta_{X}(4457)=0.099 \pm 0.012$. The branching ratio is $\mathrm{Br}_{X}=$ $0.9 \%$ also within the bounds. The photodecay widths are $\Gamma_{X \gamma}(4312)=(3.2 \pm 0.8) \times 10^{-8} \mathrm{GeV}, \quad \Gamma_{X \gamma}(4440)=$ $(1.05 \pm 0.26) \times 10^{-7} \mathrm{GeV}$, and $\Gamma_{X \gamma}(4457) \approx 0$. These observations extend to the newly predicted bottom pentaquark states in $[9,10]$.

\section{C. $\operatorname{spin} \frac{3}{2}$}

For a pentaquark state $\left[\frac{1}{2} \frac{3}{2}^{-}\right]_{S=1}$ the contributions are similar, with the bulk covariantized contribution [42]

$\tilde{\eta}_{X} \int d z d^{4} x \sqrt{|g(z)|} e^{-\phi(z)} \sum_{\xi=1,2} \bar{\psi}_{\xi} e_{A}^{M} e_{B}^{N} e_{C}^{L} \Gamma^{C} \sigma^{A B} F_{M N} \Psi_{L, \xi}$,

instead of (9). The Rarita-Schwinger propagator of the $\left[\frac{1}{2} \frac{3}{2}\right]_{S=1}$ pentaquark at the boundary is

$$
\begin{aligned}
S_{\mu \nu}^{F}= & \frac{i\left(\not p+\not k+m_{X}\right)}{s-m_{X}^{2}+i m_{X} \Gamma_{X}} \times\left[\eta_{\mu \nu}-\frac{1}{3} \gamma_{\mu} \gamma_{\nu}-\frac{1}{15 m_{X}}\left((p+k)_{\mu} \gamma_{\nu}\right.\right. \\
& \left.\left.+\gamma_{\mu}(p+k)_{\nu}\right)-\frac{8}{15} \frac{(p+k)_{\mu}(p+k)_{\nu}}{m_{X}^{2}}\right] .
\end{aligned}
$$

The net contribution to the squared s-channel amplitude without crossing is

$$
\mathcal{M}_{\frac{3}{2}} \approx e^{2} \frac{\left[\frac{\tilde{n}_{X}}{\tilde{\tilde{\kappa}}} \mathcal{I}(0,0)\right]\left[\frac{\tilde{n}_{X}}{\tilde{\tilde{\kappa}}} \mathcal{I}\left(0,-m_{V}^{2}\right)\right]}{s-m_{X}^{2}+i m_{X} \Gamma_{X}} \mathcal{A}_{\frac{3}{2}}\left(S^{\prime}, S\right)+\mathrm{cross},
$$

with 


$$
\begin{aligned}
\mathcal{A}_{\frac{3}{2}}\left(S^{\prime}, S\right)= & \bar{u}\left(p^{\prime}, S^{\prime}\right) \times \gamma^{\mu} \times\left(\not k^{\prime} \phi^{\star}-\phi^{\star} \not k^{\prime}\right) \times\left(\not p+\not k+m_{X}\right) \\
& \times\left[\eta_{\mu \nu}-\frac{1}{3} \gamma_{\mu} \gamma_{\nu}-\frac{1}{15 m_{X}}\left((p+k)_{\mu} \gamma_{\nu}\right.\right. \\
& \left.\left.+\gamma_{\mu}(p+k)_{\nu}\right)-\frac{8}{15} \frac{(p+k)_{\mu}(p+k)_{\nu}}{m_{X}^{2}}\right] \\
& \times(\not \not k-\not k \phi) \times \gamma^{\nu} \times u(p, S) .
\end{aligned}
$$

Given (44)-(45), the contribution of the $\left[\frac{1}{2} \frac{3}{2}^{-}\right]_{S=1}$ pentaquark to the differential cross section follows the same reasoning as the $\left[\frac{1}{2} \frac{1}{2}^{-}\right]_{S=0,1}$ ones. However, the detailed construction is not needed. Indeed, in the hidden channel decay analysis, we have found that all the decay amplitudes $\mathcal{M}$ are the same, after summing over the initial and final isospin and spin states. The difference in the partial decay width of the $\left[\frac{1}{2} \frac{1}{2}^{-}\right]_{S=0}$ and $\left[\frac{1}{2} \frac{3}{2}\right]_{S=1}$ pentaquarks, is caused by the spin averaging factor $\frac{1}{2 S+1}$. Therefore, for the photoproduction case where the X-pentaquark is in the intermediate state, and where all spin-isospin are summed over, the final amplitude is the same irrespective of the intrinsic spin values $S=0,1$. The kinematical differences introduce by (45) versus (31) are qualitative.

\section{ANALYSIS}

The combined contributions to the differential cross section for the photoproduction of a heavy meson $V=J / \Psi, \Upsilon$ is

$$
\left(\frac{d \sigma}{d t}\right) \approx \sum_{X}\left(\frac{d \sigma}{d t}\right)_{X}+\left(\frac{d \sigma}{d t}\right)_{G}
$$

when restricted to the lowest pentaquark states $\left[\frac{1}{2} \frac{1}{2}^{-}\right]_{S=0,1}$ and $\left[\frac{1}{2} \frac{3}{2}-\right]_{S=1}$ contributions.

In Fig. 3 we show the s-channel contribution to the differential cross section for $V=J / \Psi$ photoproduction versus $t$, including all the three pentaquark contributions.

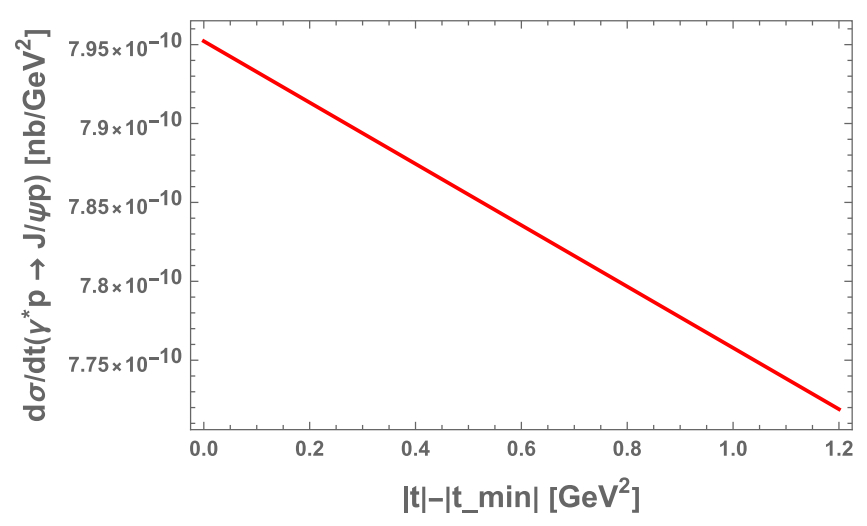

FIG. 3. s-channel contribution to the photoproduction differential cross section for $V=J / \Psi$, including all three charm pentaquark contributions.

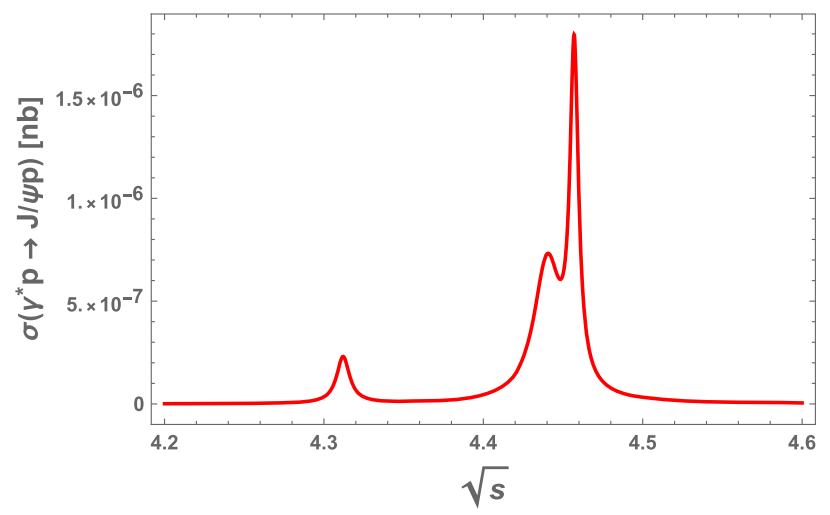

FIG. 4. s-channel contribution to the photoproduction cross section for $V=J / \Psi$ versus $\sqrt{s}$, showing the three charm pentaquarks.

In Fig. 4 we show the s-channel contribution to the total cross section for $V=J / \Psi$ photoproduction versus $\sqrt{s}$ in the threshold region, from the three pentaquarks. The smallness of the cross sections follow from the smallness of the Pauli couplings, as inferred from the holographic construction. Recall that these couplings are commensurate with the bounds on the branching ratios $[40,41]$ as we noted earlier.

The full photoproduction cross section with s- and t-channel contributions combined is shown in Fig. 5. The blue solid curve is the t-channel holographic contribution from [38], the red solid curve is our three holographic pentaquark contributions after rescaling by $\mathcal{N}_{s}=2.0 \times 10^{6}$ to make them visible. The data are from the recent results by the GlueX collaboration [40]. The smallness of the s-channel pentaquark contribution makes it equally hard to detect in the differential cross section $d \sigma / d t$ versus $|t|$ as we show in Fig. 6. The suppression is larger for the s-channel photoexcitation of bottom pentaquarks, with the extra penalty

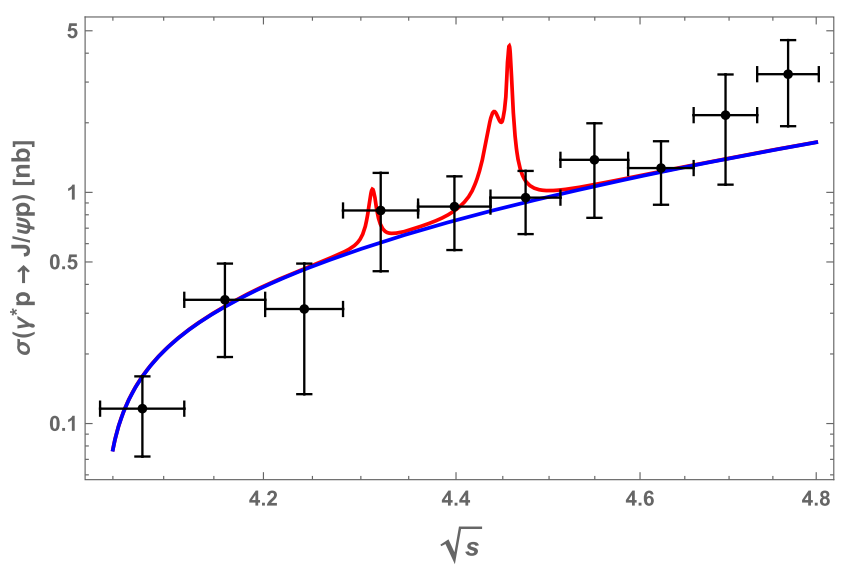

FIG. 5. Total cross section for $V=J / \Psi$ photoproduction: the blue-solid curve is the t-channel contribution from [38], the redsolid curve is the sum of t- and s-channel contribution showing the three holographic pentaquarks times $\mathcal{N}_{s}=2.0 \times 10^{6}$ to make them visible, and the data are from GlueX [40]. 


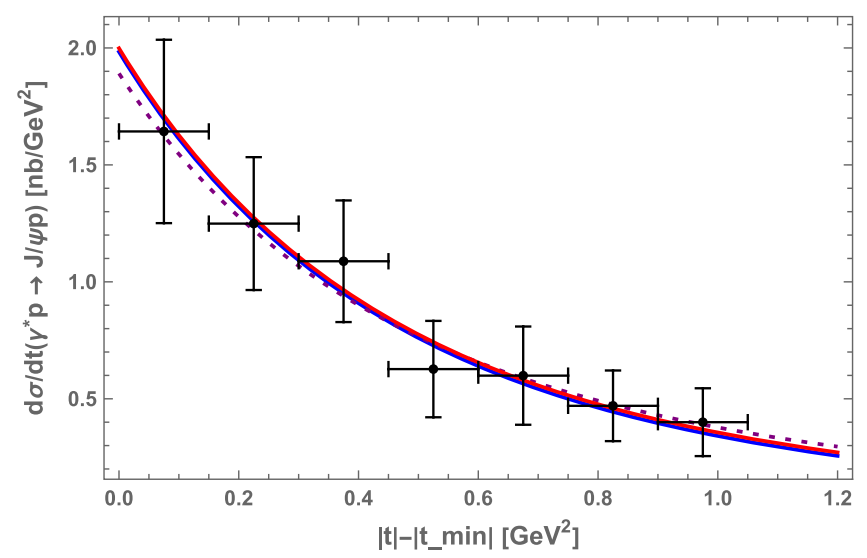

FIG. 6. s-channel plus t-channel contributions to the photoproduction differential cross section for $V=J / \Psi$, including all three charm pentaquark contributions. The blue-solid curve is the holographic t-channel contribution. The dashed-purple line is from lattice QCD using the holographic kinematic factors. The solid-red line is the total s- and t-channel contribution with the s-channel contribution multiplied by $\mathcal{N}_{s}=2.0 \times 10^{7}$ to make it slightly visible. The data are from GlueX [40] at $\sqrt{s}=4.6 \mathrm{GeV}$.

$$
\left(\frac{\eta_{P_{b}}}{\eta_{P_{c}}}\right)^{4}=\left(\frac{\mu_{P_{b}}}{\mu_{P_{c}}}\right)^{4}=\left(\frac{m_{P_{c}}}{m_{P_{b}}}\right)^{6} \approx\left(\frac{4440}{11163}\right)^{6} \approx 0.004
$$

following from (8). The t-channel contribution remains unchanged modulo kinematics.

\section{CONCLUSIONS}

The holographic construction predicts three holographic pentaquarks with the spin-isospin assignments $\left[\frac{1}{2} \frac{1}{2}^{-}\right]_{S=0,1}$ and $\left[\frac{1}{2} \frac{3}{2}-\right]_{S=1}$, which are split mostly by spin-orbit effects. It also allows for the specific evaluation of their partial decay modes with open or hidden heavy charm (bottom) most of which are yet to be measured. The holographic pentaquarks are bulk heavy-light meson fields bound to an instanton core. They are dual to a baryon core binding heavy-light mesons at the boundary with manifest chiral and heavy quark symmetry.

Given the specific spin-isospin assignments for the pentaquark states, the holographic construction allows for an explicit evaluation of their partial and total strong decay widths with open or hidden charm (bottom) in the final states. This allows for the extraction of the electromagnetic transition couplings from a proton to a pentaquark, and decay from a pentaquark. It also allows for the evaluation of the s-channel contribution to photoproduction of charmonium (bottomonium) in the threshold region. In the holographic construction, the process is mostly dominated by the t-channel graviton in bulk which is dual to a spin-2 glueball at the boundary [38].

Our results show that the t-channel contribution at threshold dwarfs the s-channel contribution by several orders of magnitude, making the current search of the charm (bottom) pentaquark states at current electron facilities out of reach. This observation appears consistent with the newly reported result by the GlueX collaboration [40], regarding the absence of a pentaquark resonance in the photoproduction of charmonium.

\section{ACKNOWLEDGMENTS}

I.Z. would like to thank Zein-Eddine Meziani for discussions. This work is supported by the Office of Science, U.S. Department of Energy under Contract No. DE-FG-88ER40388, and by the Polish National Science Centre (NCN) Grant No. UMO-2017/27/B/ST2/ 01139. K. A. M. is supported by the U.S. Department of Energy, Office of Science, Office of Nuclear Physics, contract no. DE-AC02-06CH11357, and an LDRD initiative at Argonne National Laboratory under Project No. 2020-0020.

\section{APPENDIX: $X \rightarrow V+p$ DECAY IN THE SOFT WALL MODEL}

For completeness, we show that the vectorlike interaction (7) in the (nonrelativistic) heavy mass limit, yields a decay width $X \rightarrow V+p$ in the soft wall construction, that is compatible with the one derived in the Sakai-Sugimoto model using the bound state approach $[9,10]$. For that, we note that the corresponding form factor (23) gives for the partial decay width

$$
\Gamma_{X V}=\frac{\left|\vec{q}_{V}\right|}{4 \pi m_{X}^{2}} \frac{\sum_{S, S^{\prime}}\left|\mathcal{W}\left(-m_{V}^{2}\right)\right|^{2}}{2 S+1},
$$

with $\vec{q}_{V}$ the 3-momentum of the emitted vector meson, and where $S, S^{\prime}$ refer to the spin of the initial and final states. In the heavy-quark mass and heavy-nucleon mass limit, the Pauli form factor reduces to

$$
\begin{aligned}
& \frac{\alpha_{X}}{m_{X}} \bar{u}\left(P, S^{\prime}\right)\left(\not \gamma^{\mu}-\gamma^{\mu} \not\right) u(P, S) \\
& \rightarrow \frac{\alpha_{X}}{m_{X}}\left(0, \frac{4 i}{\sqrt{m_{X} m_{N}}} \vec{q} \times \bar{u}_{S^{\prime}} \vec{\sigma} u_{S}\right)
\end{aligned}
$$

The spin source in (A2) is similar to the spin source in (3), although the latter follows by crossing from the former, hence the difference in the intrinsic parity assignment of the transition vertex. The corresponding partial decay width is

$$
\Gamma_{X V}=\left|\vec{q}_{V}\right| \times \frac{\left|\vec{q}_{V}\right|^{2}}{8 \pi m_{X}^{2}} \frac{\left(8 \alpha_{X}\right)^{2} m_{N}}{m_{X}}\left|\mathcal{I}\left(n_{X},-m_{V}^{2}\right)\right|^{2}
$$

which is to be compared with the partial decay width we obtained in the Sakai-Sugimoto construction [10] 


$$
\Gamma_{X V}=\left|\vec{q}_{V}\right| \times \frac{\left|\vec{q}_{V}\right|^{2}}{8 \pi m_{X}^{2}} \frac{\lambda^{2} m_{N}}{m_{X}}\left(\frac{M_{K K}}{m_{X}} \frac{\left\langle\varphi_{V}(0)\right\rangle^{2}}{\kappa}\right) .
$$

with $V=n=1$ for the lowest vector mode in bulk. We note that the Pauli-like coupling is the only minimal derivative term that gives a partial decay width $\Gamma \sim\left|\vec{q}_{V}\right|^{3}$ in the nonrelativistic limit. In the heavy quark limit, we can fix $\alpha_{X}$ by matching the partial widths

$$
\begin{aligned}
\alpha_{X} & =\lambda\left(\frac{\left\langle\varphi_{V}(0)\right\rangle}{8 \sqrt{\kappa}\left|\mathcal{I}\left(n_{X},-m_{V}^{2}\right)\right|}\right)\left(\frac{M_{K K}}{m_{X}}\right)^{\frac{1}{2}} \\
& =\lambda C_{V}\left(\frac{m_{N}}{m_{X}}\right)^{\frac{1}{2}} .
\end{aligned}
$$

[1] Roel Aaij et al. (LHCb Collaboration), Observation of a Narrow Pentaquark State, $P_{c}(4312)^{+}$, and of Two-Peak Structure of the $P_{c}(4450)^{+}$, Phys. Rev. Lett. 122, 222001 (2019).

[2] Roel Aaij et al. (LHCb Collaboration), Observation of $J / \psi p$ Resonances Consistent with Pentaquark States in $\Lambda_{b}^{0} \rightarrow J / \psi K^{-} p$ Decays, Phys. Rev. Lett. 115, 072001 (2015).

[3] Yizhuang Liu and Ismail Zahed, Heavy baryons and their exotics from instantons in holographic QCD, Phys. Rev. D 95, 116012 (2017).

[4] Yizhuang Liu and Ismail Zahed, Heavy and strange holographic baryons, Phys. Rev. D 96, 056027 (2017).

[5] Ming-Zhu Liu, Ya-Wen Pan, Fang-Zheng Peng, Mario Sánchez Sánchez, Li-Sheng Geng, Atsushi Hosaka, and Manuel Pavon Valderrama, Emergence of a Complete Heavy-Quark Spin Symmetry Multiplet: Seven Molecular Pentaquarks in Light of the Latest LHCb Analysis, Phys. Rev. Lett. 122, 242001 (2019).

[6] C. W. Xiao, J. Nieves, and E. Oset, Heavy quark spin symmetric molecular states from $\bar{D}^{(*)} \Sigma_{c}^{(*)}$ and other coupled channels in the light of the recent LHCb pentaquarks, Phys. Rev. D 100, 014021 (2019).

[7] Meng-Lin Du, Vadim Baru, Feng-Kun Guo, Christoph Hanhart, Ulf-G. Meißner, José A. Oller, and Qian Wang, Revisiting the nature of the $P_{c}$ pentaquarks, J. High Energy Phys. 08 (2021) 157.

[8] Mao-Jun Yan, Fang-Zheng Peng, Mario Sánchez Sánchez, and Manuel Pavon Valderrama, Interpretations of the new LHCb $P_{c}(4337)^{+}$pentaquark state, arXiv:2108.05306.

[9] Yizhuang Liu, Maciej A. Nowak, and Ismail Zahed, this issue, Holographic charm and bottom pentaquarks. I. Mass spectra with spin effects, Phys. Rev. D 104, 114021 (2021).

[10] Yizhuang Liu, Maciej A. Nowak, and Ismail Zahed, preceding paper, Holographic charm and bottom pentaquarks. II. Open and hidden decay widths, Phys. Rev. D 104, 114022 (2021).

[11] Roel Aaij et al. (LHCb Collaboration), Evidence for a new structure in the $J / \psi p$ and $J / \psi \bar{p}$ systems in $B_{s}^{0} \rightarrow J / \psi p \bar{p}$ decays, arXiv:2108.04720.

[12] M. B. Voloshin and L. B. Okun, Hadron molecules and charmonium atom, JETP Lett. 23, 333 (1976).
[13] Marek Karliner and Jonathan L. Rosner, New Exotic Meson and Baryon Resonances from Doubly-Heavy Hadronic Molecules, Phys. Rev. Lett. 115, 122001 (2015).

[14] Richard F. Lebed, Ryan E. Mitchell, and Eric S. Swanson, Heavy-quark QCD exotica, Prog. Part. Nucl. Phys. 93, 143 (2017).

[15] A. Esposito, A. Pilloni, and A. D. Polosa, Multiquark resonances, Phys. Rep. 668, 1 (2017).

[16] Stephen Lars Olsen, Tomasz Skwarnicki, and Daria Zieminska, Nonstandard heavy mesons and baryons: Experimental evidence, Rev. Mod. Phys. 90, 015003 (2018).

[17] Feng-Kun Guo, Christoph Hanhart, Ulf-G. Meißner, Qian Wang, Qiang Zhao, and Bing-Song Zou, Hadronic molecules, Rev. Mod. Phys. 90, 015004 (2018).

[18] Marek Karliner, Jonathan L. Rosner, and Tomasz Skwarnicki, Multiquark states, Annu. Rev. Nucl. Part. Sci. 68, 17 (2018).

[19] Rui Chen, Xiang Liu, Xue-Qian Li, and Shi-Lin Zhu, Identifying Exotic Hidden-Charm Pentaquarks, Phys. Rev. Lett. 115, 132002 (2015).

[20] Giancarlo Rossi and Gabriele Veneziano, The string-junction picture of multiquark states: An update, J. High Energy Phys. 06 (2016) 041.

[21] Michael I. Eides, Victor Yu. Petrov, and Maxim V. Polyakov, Pentaquarks with hidden charm as hadroquarkonia, Eur. Phys. J. C 78, 36 (2018).

[22] Feng-Kun Guo, Hao-Jie Jing, Ulf-G Meißner, and Shuntaro Sakai, Isospin breaking decays as a diagnosis of the hadronic molecular structure of the $P_{c}(4457)$, Phys. Rev. D 99, 091501 (2019).

[23] Hua-Xing Chen, Wei Chen, and Shi-Lin Zhu, Possible interpretations of the $P_{c}(4312), P_{c}(4440)$, and $P_{c}(4457)$, Phys. Rev. D 100, 051501 (2019).

[24] Rui Chen, Zhi-Feng Sun, Xiang Liu, and Shi-Lin Zhu, Strong LHCb evidence supporting the existence of the hidden-charm molecular pentaquarks, Phys. Rev. D 100, 011502 (2019).

[25] Michael I. Eides, Victor Yu Petrov, and Maxim V. Polyakov, New LHCb pentaquarks as hadrocharmonium states, Mod. Phys. Lett. A 35, 2050151 (2020).

[26] N. N. Scoccola, D. O. Riska, and Mannque Rho, Pentaquark candidates $P_{c}^{+}(4380)$ and $P_{c}^{+}(4450)$ within the soliton picture of baryons, Phys. Rev. D 92, 051501 (2015). 
[27] Jacob Sonnenschein and Dorin Weissman, A tetraquark or not a tetraquark? A holography inspired stringy hadron (HISH) perspective, Nucl. Phys. B920, 319 (2017).

[28] Hans Gunter Dosch, Guy F. de Teramond, and Stanley J. Brodsky, Supersymmetry across the light and heavylight hadronic spectrum, Phys. Rev. D 92, 074010 (2015).

[29] Yong-Hui Lin and Bing-Song Zou, Strong decays of the latest $\mathrm{LHCb}$ pentaquark candidates in hadronic molecule pictures, Phys. Rev. D 100, 056005 (2019).

[30] Qian Wang, Xiao-Hai Liu, and Qiang Zhao, Photoproduction of hidden charm pentaquark states $P_{c}^{+}(4380)$ and $P_{c}^{+}$(4450), Phys. Rev. D 92, 034022 (2015).

[31] V. Kubarovsky and M. B. Voloshin, Formation of hiddencharm pentaquarks in photon-nucleon collisions, Phys. Rev. D 92, 031502 (2015).

[32] Marek Karliner and Jonathan L. Rosner, Photoproduction of exotic baryon resonances, Phys. Lett. B 752, 329 (2016).

[33] Z. E. Meziani et al., A search for the $\mathrm{LHCb}$ charmed 'pentaquark' using photo-production of $J / \psi$ at threshold in hall C at Jefferson Lab, arXiv:1609.00676.

[34] Tadakatsu Sakai and Shigeki Sugimoto, Low energy hadron physics in holographic QCD, Prog. Theor. Phys. 113, 843 (2005).
[35] A. Gorsky, P. N. Kopnin, and A. Krikun, Baryon as dyonic instanton-II. Baryon mass versus chiral condensate, Phys. Rev. D 89, 026012 (2014).

[36] Deog Ki Hong, Mannque Rho, Ho-Ung Yee, and Piljin Yi, Dynamics of baryons from string theory and vector dominance, J. High Energy Phys. 09 (2007) 063.

[37] Kiminad A. Mamo and Ismail Zahed, Neutrino-nucleon DIS from holographic QCD: PDFs of sea and valence quarks, form factors, and structure functions of the proton, Phys. Rev. D 104, 066010 (2021).

[38] Kiminad A. Mamo and Ismail Zahed, Diffractive photoproduction of $J / \psi$ and $\Upsilon$ using holographic QCD: Gravitational form factors and GPD of gluons in the proton, Phys. Rev. D 101, 086003 (2020).

[39] Kiminad A. Mamo and Ismail Zahed, Electroproduction of heavy vector mesons using holographic QCD: From near threshold to high energy regimes, Phys. Rev. D 104, 066023 (2021).

[40] A. Ali et al. (GlueX Collaboration), First Measurement of Near-Threshold $J / \psi$ Exclusive Photoproduction off the Proton, Phys. Rev. Lett. 123, 072001 (2019).

[41] Xu Cao and Jian-ping Dai, Confronting pentaquark photoproduction with new LHCb observations, Phys. Rev. D 100, 054033 (2019).

[42] Eric D'Hoker and Bijan Pourhamzeh, Emergent superVirasoro on magnetic branes, J. High Energy Phys. 06 (2016) 146. 